\title{
From Command to Control: interface design for customer handling systems
}

\author{
Nicola Millard*, Linda Hole ${ }^{*}$ \& Simon Crowle \\ "BT Laboratories, Martlesham Heath, Ipswich, England ${ }^{1}$ \\ "Bournemouth University, Wallisdown, Bournemouth, England \\ nicola.millard@bt-sys.bt.co.uk \\ lhole@bournemouth.ac.uk \\ scrowle@bournemouth.ac.uk
}

\begin{abstract}
This paper describes interface design activity which focused on the tasks performed by call centre advisers. The advisers' work is supported by a large database, which offers them a command line interface. Contextual observation revealed that more effort was needed to interrogate the database than to communicate with the customers. A subset of advisers' tasks was used to provide scenarios for the design of a 2D/3D graphical user interface, which was prototyped in Macromind Director ${ }^{\mathrm{TM}}$ and evaluated by a user group. Initial feedback indicated that the design metaphor and dialogue style have the potential to radically improve the advisers' interaction with the system.
\end{abstract}

KEY WORDS case study database GUI design

\section{INTRODUCTION}

Systems which support the telephone interaction between a customer adviser and their customers in a call centre environment present numerous challenges to the user interface designer. These user interfaces need to:

- present a large amount of complex information;

- present the right information at the right place at the right time in the right format;

- provide the customer adviser with a navigation mechanism that does not distract them from their conversation with the customer;

- provide a coherent, efficient and effective method for getting information to the customer at their first point of contact.
Typically, customer service centres provide their customer service advisers with a command line interface to these systems. These tend to fulfil the first criteria but are difficult to use, require a high commitment for training and place a significant cognitive load onto the customer adviser, thus distracting them from their principle task: talking to the customer. A forms driven interface is also common in customer service centres and solves some of these problems. This research was initiated to investigate whether a prototype graphical user environment could be introduced into an operational service centre and fulfil these criteria. The prototype was intended to provoke discussion among systems designers as to the viability of introducing such interfaces into

\footnotetext{
1 Thanks are due to Mike Ward of BT Laboratories and the staff of the BT Customer Service Centre, Bournemouth, for their support and encouragement throughout the project.
} 
operational environments. The research team was given access to a customer service centre in the South of England taking incoming calls on a wide range of topics relating to their telephony services.

The study was carried out in three phases:

1. observation of the advisers working with the command line interface;

2. design of the graphical user interface;

3. evaluation of the new interface.

\section{PHASE ONE: PROBLEM IDENTIFICATION}

Phase one of the study provided an overview of the use of the customer service database to:

- show how the system represented the requirements of the advisers' tasks;

- demonstrate the methods the advisers used to perform those tasks;

- relate the methods used to performance of the task itself.

The research team needed to obtain a users' (adviser's) view of the command language access to the database so that a true picture of call handling activity could be developed. The study began with passive observation of the customer advisers' interaction with the database within their work context. A total of 365 calls were observed with 11 advisers as they performed the tasks of receiving and responding to customer enquiries. During the call, the advisers performed actions such as: browsing the database; writing a note; looking for information off-line. The data consisted of sequences of the command language codes used to browse the database during each call, plus any other actions the advisers performed during the call.

Three call scenarios were identified during analysis: bill enquiries; features sales; moving house. Actions were collated and analysed for 180 calls which fell within the three scenarios. It was found that within each scenario, $83 \%$ of adviser activity was focused on a central 16 typical actions. A task flow analysis was obtained from the bank of taped verbal protocols of customer calls. Approximately $10 \%$ of the calls in each category were transcribed from the tapes to produce an activity flow, illustrating how the conversation between customer and adviser mapped onto the adviser's work with both the system and off-line facilities.

The findings from the task analysis were as follows:
1. Only a comparatively small subset of database screens were used during call handling, and, within the subset, activity was primarily focused on a few key screens.

2. Movement between the screens did not follow the prescribed menu-driven route: the experienced advisers had learnt the three-letter commands to access the area of the database they required.

3. Interaction with the database in this fashion sometimes caused problems with the saving of entries in certain fields. Hence a lot of note-taking activity was required for the advisers to maintain the information they had collected from the customer or their records.

4. Information presented on a screen was sometimes needed in conjunction with other screens. This added to the users' cognitive load as they were required to flick backwards and forwards between screens to follow their thoughts about the customer enquiry.

5. The advisers rarely used the system menus or help facilities; when they forgot how to access areas of the database, they relied on one another for help.

6. Customers' personal data were repeatedly noted, whereas the system might have been expected to carry that data throughout the interaction.

7. Accessing the database via this interface meant that a disproportionate number of actions were required to complete each task.

Passive observation was chosen as a method which would not disrupt the advisers' natural work pattern. However, it did mean that none of the advisers' views could be elicited during data collection. A questionnaire was administered to 30 advisers to ascertain their overall impression of the database; approximately $43 \%$ were returned for analysis. The findings indicated that:

1. Advisers suggested that the system was 'easier' rather than more 'difficult' to use. A possible explanation of this could be the experience of the users, none of whom could be classified as a novice or intermediate user. Since it is the nature of the job that advisers should be competent in their knowledge and use of the system, it is not unreasonable for persons of this level to consider the job 'easy'.

2. Although the advisers found the database adequate for their jobs, they felt that the system was sometimes a hindrance to them doing the job to the best of their abilities. 
3. The single largest area of concern was the speed of interaction required to meet the call handling time, which was targeted at 240 seconds per telephone call.

4. Many advisers enjoyed their jobs and took a pride in successfully and efficiently dealing with the customer enquiries and problems. They basically viewed themselves as the database access provider for the customer, mastering the interface on the customer's behalf

The database provided all the information that the advisers could ever need, although it wasn't necessarily on one screen. Its command line interface relied heavily upon fast and accurate typing. Interaction with the system was powerful, flexible, and user controlled: experienced advisers could access the information held within the database with speed and efficiency; they could gain instant access to all areas of the database within their level of authorisation. However, this interface made a heavy demand on the adviser's long term memory to recall the three letter screen commands, making the system difficult to learn and to remember. The reliance upon typed input made the use of the system error prone as the advisers attempted to work at a speed which enabled them to meet their call handling targets. The advisers did not always work in the manner required by the system, and hence they had to undertake lots of notetaking as parameters were lost from screen to screen. During a call, the advisers browsed the database to gain an understanding of the customer's circumstances as they were speaking, holding information from the various locations in their working memory.

\section{PHASE TWO: INTERFACE DESIGN} Phase two generated the prototype interface, POW (Personal Office Workspace), which was a result of:

- addressing some important $\mathrm{HCI}$ issues that are problematic in command-language interfaces;

- reviewing actions the advisers took to perform the three scenarios;

- synthesising modern interface metaphors and interaction styles with the advisers' activities.

\subsection{Scenario based design}

A scenario-based approach looks at the user, their environment and the context of the work. In the particular case of these customer advisers, the customer exists as part of the scenario and the interaction between the adviser and the customer (not just the adviser and the system) must also be considered (Carroll, 1995). The design of the interface focused on the advisers, their work and how the system could most effectively support their activities. The functionality was defined in terms of adviser activities, the information requirements for those activities and how the activities supported the advisers' work. Developing a system using scenarios allowed both the designers and advisers to communicate using a common point of reference (the office environment).

Prototyping from scenarios begins with a relatively simple model of the system. Users test the prototype with real-world work scenarios suggested by both the designer and user. Using this method, the designer and user can quickly pick up errors in design, identify areas that need development and capture usability problems.

\subsection{System analysis}

POW's 3D, graphical user interface reduces cognitive load and provides user-controlled methods of interaction. For example, users need only recognise information sources (Phonebook, Streetfinder, etc) rather than having to recall them (3-letter command codes), and the objects offer affordances (Norman, 1988) which inform the users about their potential behaviour. The system also provides some automation (with automatic retrieval of telephone numbers) and alleviates the need to note system status.

Information is presented in a more manageable and meaningful way; POW objects are taken from real-world scenarios that both customer and adviser are familiar with and that encapsulate information in a recognisable format (eg presenting billing information as the customer sees it). This graphical ad hoc manipulation interface (Kappel \& Min Tjoa, 1992) enables the adviser to manipulate the environment to carry out a task. By using direct manipulation interface metaphors to access/process information, POW allows its users to concentrate on what they are doing rather than how they are doing it (Weller \& Hartson, 1992).

\subsection{Interface design}

In designing the interface, the team took a craft approach, relying on the three scenarios to guide the design decisions. Lansdale, Scrivener and Woodcock (1996) comment that in $\mathrm{HCI}$, disciplines such as task analysis or cognitive psychology are insufficiently 
developed to prescribe exactly how design should proceed in any given situation. Wallace and Anderson (1993) suggest there is a need to synthesise the sources of interface design quality: talent from the craft approach; principles from the cognitive engineering approach.

The prototype interface addressed the problems of:

- maintaining the power and flexibility of access offered by the database, to provide an interface which would still offer the advisers control and flexibility in their work;

- removing the need to flick backwards and forwards from one screen to another: the information scattered across numerous screens needed to be provided on one screen without overloading the screen;

- ensuring that data entries were saved when moving from one information source in the database to another;

- avoiding hand-written notes which duplicated information held on the system.

In order to maintain the powerful interaction with the database which was provided via the command line interface, a graphical, direct manipulation interface was chosen: this would be flexible, easy to learn and remember, and less error prone because far less keyboard entry would be required (Mayhew, 1992). Just as the command line dialogue enabled the advisers to travel anywhere in the database, so the graphical interface would provide direct access to all of the stored data via objects on the screen. It offered easily reversible actions, instant, visual feedback, and exploited the use of visuospatial cues, to utilise recognition rather than recall memory.

The provision of a GUI shifted some of the advisers' cognitive load to their perceptual system. Rather than keeping the task requirements within their working memory, the advisers would be able to obtain visual cues from the objects on the screen to remind them which procedures they had/had not completed. The declarative actions using semantic memory in the command line dialogue were replaced with procedural actions using episodic memory in the direct manipulation dialogue (Anderson, 1980). Wright and Lickorish (1994) observed that in any complex task that makes many demands on memory processes, people will select procedures that result in fewest additional demands on their working memory.

The proposed new interface built upon the desktop metaphor which users may already know and expect to find (Sawyer \& Mariani, 1995): it transformed the desktop into a cubicle. This provided the customer advisers with a visual image of their own, virtual, 3D workspace, enclosed by "walls" which could be enhanced by the adviser's own personal pictures, notes, etc. The cubicle, at desktop level, housed a variety of objects the adviser needed to perform the call handling tasks.

"Books" of information provided pages of data on the same screen without the advisers having to change screens or refresh screens. The advisers' knowledge or intuition about the contents of these "books" would reduce the need to hold the numerous command codes in their memories. Data was shown to be stored 'within' the objects and was consistent with the adviser's conception of information storage and retrieval with these objects in everyday life.

"Peel-off notes" took the role of information holders; in everyday life we jot things on them and move them around, so why not transfer this process to the screen? Data was manipulated by the use of these "peel-off notes", which could be: written on and dragged to a file to instigate a search for the customer record; peeled off record $A$ and dragged to record $B$ to effect an update of the customer records; written on and stuck onto a customer's page to annotate the customer record.

\section{PHASE THREE: INTERFACE EVALUATION}

The POW prototype, based on the three call handling scenarios, was engineered with a small amount of data to enable some data manipulation to take place. During phase three of the study, POW was shown to a new group of twenty customer advisers to assess their initial reactions to the interface. Individual interviews were conducted to obtain detailed views from users who were of varied experience.

Prior to seeing POW for the first time, the advisers were encouraged to focus their thoughts on the customer service database interface currently used by means of a brief questionnaire. The questionnaire presented strengths and weaknesses of the command language dialogue style (Mayhew, 1992) to elicit the advisers' judgements of how their system measured up against these criteria. This was an attempt to highlight the power of the command language interface, so that the advisers might take a more critical view of the direct manipulation interface, rather than being swayed by its novelty. 
The interviews were video-recorded to collect all the advisers' interaction on screen, plus their comments. The interviews were used to establish the adviser's: previous computer experience; interpretations of the system metaphor; expectations of the interactive objects displayed on screen; reactions and comments regarding system design. All participants were informed before the session began that their interview was strictly confidential and that it was not a test of intelligence or aptitude. The video camera was pointed at the computer monitor, not at the participant.

\subsection{Results}

The questionnaire data confirmed some of the phase one findings from the first group of advisers. There was overall disagreement that the command line interface to the customer service database:

- was fast and efficient;

- was flexible;

- offered screens which were consistent in appearance;

- allowed the users to customise the screens;

- allowed the users to enter multiple commands;

- trapped errors;

- allowed the users to undo any erroneous actions.

There was a 50/50 divide of opinion that the customer service database:

- was easy to learn;

- enabled them to enter and exit the screens as they wished;

- offered screens which were well designed.

There was overall agreement that the customer service database:

- was clear and easy to understand;

- used the keyboard as a satisfactory input device;

- allowed access to customer information;

- allowed the user to check input before it was actioned;

- provided a useful HELP facility.

From the open-ended questions it was apparent that:

- the best aspect of their current system was the sense of power the advisers experienced by having the ability to access information directly, and by being familiar with the system;

- the worst aspects were both design and performance related: the customer service database was considered to be slow, cumbersome, unreliable, complex, inflexible, unclear and suffered from a lack of prompts;
- some advisers felt the system relied too heavily on jargon, and the information provided on-screen was difficult to understand or use;

- the most common mistakes that they encountered were due to typing errors, incorrect input and general systems errors.

The advisers suggested that the design of the customer service database could be improved by: less typing; reordering of the information with an emphasis on keeping the relevant information together and having direct access to that information; an error correction facility; fewer screens; having a list of customer options; having the ability to page back and forward without having to save information; increasing the speed between screens to allow quicker access to frequently used screens.

The interview data was organised into a set of keywords or phrases describing:

a) initial adviser reaction to aspects of the system;

b) in-depth comments on aspects of the system;

c) features advisers would like to see added to/changed in the system;

d) how the advisers would handle the three call scenarios with the system.

It was interesting to note the wide variety of abilities amongst the advisers. Those who had gained previous experience with direct manipulation system metaphors were more capable of grasping the concepts behind the interface and expressing their expectations of the system. Those with no previous computer experience and/or who had used the customer service database for many years appeared to try to interpret the new system in terms of the current system's functions. However, almost without exception the reception to the new system was positive and very encouraging.

$65 \%$ of the advisers recognised the whole environment to be a representation of an office or a room. Others broke the scenario down into smaller components such as a filing cabinet, or a shelf of books. Some of the more literal minded described it as an 'integrated database' or '3D outlines' (of books).

$35 \%$ of the advisers guessed that the "peel-off-note" was something to do with accessing the customerís details; $25 \%$ of advisers thought it to be a page from one of the books, a diary or a piece of paper; $30 \%$ could not identify it; $10 \%$ could not actively use the system (could not use the mouse), they only gave comments. 
The prototype caused one or two interaction difficulties (eg closing the books and putting away the flipchart), and some advisers said that it 'would take a bit of getting used to'. Despite these problems, the overwhelming response to the interface was encouraging.

How the advisers would handle the three call scenarios with the system was categorised in terms of the use of an object (or objects) on screen. Some advisers were able to elaborate on how they would use the information from each object within the context of a) the scenario and b) the system as a whole, whilst others could only identify which objects they would use to achieve their goal. All advisers had little or no difficulty in proposing a solution to the call handling scenarios using the system, which suggests that they had become engaged in the POW environment (Weller \& Hartson, 1992).

\section{DISCUSSION}

Testing the POW prototype and receiving user feedback was an essential part of the development strategy. By analysing the video recordings of the interviews it was possible to determine how the POW objects matched up to the advisers' expectations; for example which "book" the adviser expected to contain what information. An interaction study shows how users most commonly attempt to use the system (such as methods of opening and closing books). Also, discussions with the advisers during and after using the system revealed areas that need development (moving house using the Streetfinder) and individual differences in working practice. Interestingly, exposure to the new interface promoted active thinking on the part of the advisers, who frequently suggested new features that would help with their work. For example, one adviser suggested including spatial information on customers' addresses.

POW incorporates the best of the customer service database system whilst making considerable $\mathrm{HCI}$ progress, but still has many steps to make before it can become a reality. However, even in the first prototype stage, POW has received a positive response from the advisers.

Call centres have been established within a wide variety of organisations, each of which aim to provide a fast, friendly and efficient customer response. The customer advisers handling the incoming calls are under pressure to present a warm and co-operative human interface whilst possibly wrestling with a sterile and unyielding systems interface. If they are provided with an interface which closely resembles the real world, the advisers will feel supported by a system which more naturally maps on to the focus of their work: cooperating with their customers to solve problems.

Whilst the design of these empowering environments (Weller and Hartson, 1992) should become the focus of serious endeavour in order to release the advisers from the stress of less usable interfaces, it would be useful to also examine the possibility of enabling the advisers to enjoy their customer handling work: Norman (1986) gives this as his second goal for cognitive engineering. If the advisers find themselves perceiving their use of the computer system as fun (Igbaria, Schiffman \& Wieckowski, 1994), then their call handling behaviour will change to produce a better level of interaction with their customers.

Only $22 \%$ of the companies (of $100+$ employees) surveyed by the Henley Centre (The Henley Centre, 1994) appeared to have the appropriate level of investment in technology, telecoms services, customer research and human resources to enable them to perform an effective customer handling function. $60 \%$ of the major organisations contacted could not access their customer data either quickly or easily. $9 \%$ of companies were classed by the Henley Centre as 'advanced call handlers'. These companies are beginning to recognise that effective user interface design is the way to fulfil their complex information management needs as they interact with the customer.

A large percentage of these 'advanced call handlers' are currently using systems which have command line interfaces. Use of forms based and Windows based interfaces are more common in companies who have not had large legacy systems to integrate into their customer service processes. Use of more innovative GUI environments are rare. However, development using tools which support graphical environments are becoming more common. These are beginning to change the standard forms based look and feel of the screen environment. 


\section{CONCLUSION}

The role that user interface design plays in managing the critical interaction between a customer adviser and their customer is significant. The initial evaluation of the POW metaphor indicated that the location of commonly accessed items of customer data can be successfully represented in a graphical environment. Recognition of the elements within the POW environment made it easy to navigate and use without placing a significant amount of additional cognitive load on the customer adviser whilst interacting with the customer. Although the POW prototype was never intended to become an operational system, elements, such as the "peel off notes", have already been incorporated into operational customer handling interfaces.

The ability of the POW prototype to handle the high complexity, high volume amounts of customer data which occur in an operational environment has yet to be proven. When dealing with low level information which is less frequently accessed, a command line system is often more powerful and the POW metaphor may not be appropriate. Other issues which require further investigation are the effect of user experience on navigation and the practicality of a mouse driven user interface over keyboard driven or touchscreen in an operational environment.

Customer handling, however, is not just about innovative technologies. Peters (1989) stated that "service equals systems plus smiles". Technology can take the emphasis on process away from the human adviser so that they can concentrate on customer care. However, deskilling the adviser can take away some of the challenge and motivation of what can be a boring and repetitive task. Giving the adviser a good working environment, a supportive team and responsibilities for running the customer service centre and taking calls can all be motivating factors that promote delivery of a world class customer interface. Using the principles established in the design of the POW interface, the user's personal screen world can be used to complement and reflect the overall design of their working environment.

\section{REFERENCES}

Anderson, J R (1980) Cognitive Psychology and its Implications San Francisco: W H Freeman \& Co

Carroll, J M (1995) Scenario-Based Design: Envisioning Work \& Technology in System Development Wiley

Igbaria, M, Schiffman, S \& Wieckowski, T (1994) The respective roles of perceived usefulness and perceived fun in the acceptance of microcomputer technology Behaviour \& Information Technology, vol 13, no 6, pp349-361

Kappel, G \& Min Tjoa, A (1992) State of art and open issues on graphical user interfaces for object-oriented database systems Information \& Software Technology, vol 34, no 11, pp 721-730

Lansdale, M W, Scrivener, S A R \& Woodcock, A (1996) Developing practice with theory in $\mathrm{HCI}$ : applying models of spatial cognition for the design of pictorial databases Int. J. Human-Computer Studies, 44, pp777-799

Mayhew, D (1992) Principles \& Guidelines in Software User Interface Design London: Prentice-Hall

Norman, D (1986) 'Cognitive engineering' in Norman, D \& Draper, S (eds) User Centred System Design Lawrence Erlbaum Associates

Norman, D (1988) The Psychology of Everyday Things New York: Basic Books

Peters, T (1989) The Customer Revolution BBC Video

Sawyer, P \& Mariani, J (1995) Database systems: challenges and opportunities for graphical $\mathrm{HCI}$ Interacting with Computers, vol 7, no 3, pp273-303

The Henley Centre (1994) Teleculture 2000, July

Wallace, M D \& Anderson, T J (1993) Approaches to interface design Interacting with Computers, vol 5, no 3, pp259-278

Weller, HG \& Hartson, HR (1992) Metaphors for the Wright, P \& Lickorish A (1994) Menus and memory load: navigation strategies in interactive search tasks Int. J. Human-Computer Studies, 40, pp965-1008

Nature of Human-Computer Interaction in an Empowering Environment: Interaction Style Influences the Manner of Human Accomplishment Computers In Human Behaviour, vol 8, pp313-33 